EPJ Web of Conferences 41, 05045 (2013)

DOI: $10.1051 /$ epjconf/20134105045

(C) Owned by the authors, published by EDP Sciences, 2013

\title{
Photoinduced Processes in Cobalt-Complexes: Condensed Phase and Gas Phase
}

\author{
F. Rupp ${ }^{1}$, K. Chevalier ${ }^{1}$, M. M. N. Wolf ${ }^{1}$, H. -J. Krüger ${ }^{2}$, C. v. Wüllen ${ }^{2}$, Y. Nosenko ${ }^{2}$, G. Niedner- \\ Schatteburg $^{2}$, C. Riehn ${ }^{2}$ and R. Diller ${ }^{1}$ \\ ${ }^{1}$ Dept of Physics, Univ. Kaiserslautern, 67663 Kaiserslautern, Germany \\ ${ }^{2}$ Dept. of Chemistry, Univ. Kaiserslautern, 67663 Kaiserslautern, Germany
}

\begin{abstract}
Femtosecond time-resolved, steady-state spectroscopic methods and quantum chemical calculations are employed to study ultrafast photoinduced processes in $\left[\mathrm{Co}(\mathrm{III})-\left(\mathrm{L}-\mathrm{N}_{4} \mathrm{Me}_{2}\right)(\mathrm{dbc})\right]\left(\mathrm{BPh}_{4}\right)$ and $\left[\mathrm{Co}(\mathrm{II})-\left(\mathrm{L}-\mathrm{N}_{4} t \mathrm{Bu}_{2}\right)(\mathrm{dbsq})\right]\left(\mathrm{B}\left(\mathrm{p}-\mathrm{C}_{6} \mathrm{H}_{4} \mathrm{Cl}\right)_{4}\right)$ and to characterise the transient redox- and spin-states in condensed and gas phase.
\end{abstract}

\section{Introduction}

Metal complexes undergoing valence tautomerism (VT) and spin-crossover (SCO) by external stimuli may be utilized as novel molecular switches[1]. Here we study ultrafast photoinduced processes in the first cobalt(II) semiquinonate complex [Co(II)-(L- $\left.\left.\mathrm{N}_{4} t \mathrm{Bu}_{2}\right)(\mathrm{dbsq})\right]\left(\mathrm{B}\left(\mathrm{p}-\mathrm{C}_{6} \mathrm{H}_{4} \mathrm{Cl}\right)_{4}\right)$ (Cpd2) that displays SCO properties rather than VT, and for comparison in a related cobalt(III)

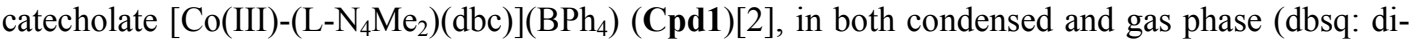
tert-butylsemiquinonate $(\mathrm{Sq})$, dbc: di-tert-butylcatecholate (Cat)). In combination with quantum chemical calculations this approach allows to follow and characterize transient valence states and molecular structure, spin states[3,4], magnetization[5], energy flow, fragmentation patterns as well as the impact of solute/(micro-) solvent interaction.

\section{Condensed phase: Femtosecond time-resolved pump-probe- spectroscopy}

After photoexcitation of $\mathbf{C p d} 2$ dissolved in acetonitrile- $\mathrm{d}_{3}$ at $1075 \mathrm{~nm}$ we probe in the visible/nearinfrared (data not shown) and mid-IR range (Figure 1 (top)) for dynamical and structural information on the transient species[3]. A bi-exponential fit of the transient absorption yields two time constants $\tau_{1} \approx 1$ ps and $\tau_{2} \approx 9$ ps. During the photocycle an electron of the Co(II) atom of $\mathbf{C p d} 2$ is suggested to be transferred to the Sq coligand due to a metal-to-ligand-charge-transfer (MLCT) that leads to the formation of the Co(III)-Cat species with life times $\tau_{1}$ and $\tau_{2}$, respectively, but different spin multiplicity. The negative net signals in the transient difference spectra in Figure 1 (top) clearly match the peak positions in the ATR-spectrum of Cpd2 (solid) in Figure 1 (middle) and show the bleach of the ground state in the mid-IR at several band positions, e.g. $1176 \mathrm{~cm}^{-1}$. The calculated stick spectrum (B3LYP, disp.-corr., TZVP basis, scaled 0.98) in Figure 1 (middle) clearly reproduces the experimental ATR-spectrum. The strong positive net signal around $1450 \mathrm{~cm}^{-1}$ indicates the formation of the intermediate Cat species, taking into account that the absorption of the 


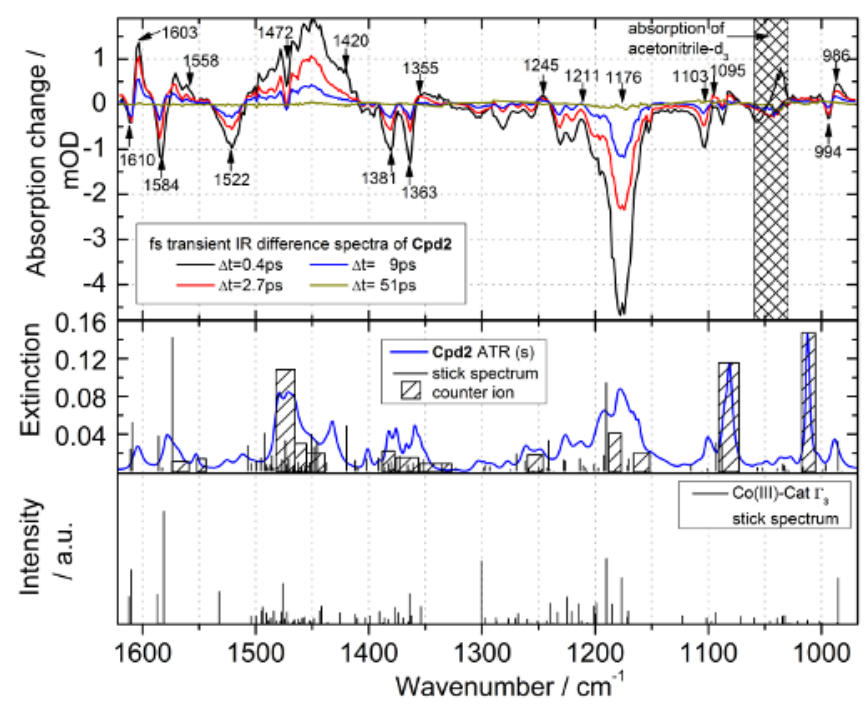

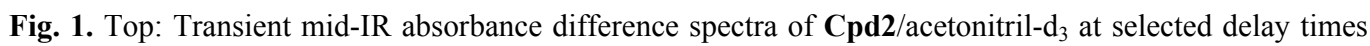
between $0.4 \mathrm{ps}$ (strong signal) and $51 \mathrm{ps}$ (weak signal) after photo-excitation at $1075 \mathrm{~nm}$ and room temperature. Middle: For comparison steady state ATR-spectrum (solid) of Cpd2 with calculated stick spectrum. Bottom: Calculated spectrum of $\mathrm{Co}$ (III)-Cat. Solvent and counter ion contributions shaded.

diamagnetic Cpd1 is significantly higher around $1450 \mathrm{~cm}^{-1}$ (data not shown). On grounds of the femtosecond time-resolved and steady state measurements, as well as the quantum chemical calculations, the following reaction scheme is suggested: After the photoexcitation of Cpd2 the system relaxes with less than 200 fs (time-resolution) into two Cat states, $\Gamma_{2}$ and $\Gamma_{3} . \Gamma_{3}$ is supposed to be a diamagnetic $\mathrm{Co}(\mathrm{III})-\mathrm{Cat}$ with $\mathrm{S}=0$ and life time $\tau_{2} \approx 9 \mathrm{ps}$ (cf. Figure 1 (bottom)) and $\Gamma_{2}$ to be a $\mathrm{Co}$ (III)-Cat, but with $\mathrm{S}=1$ and life time $\tau_{1} \approx 1$ ps. The ground state of $\mathbf{C p d} 2$ is a ferromagnetically coupled triplet state with $S=1$. Therefore the spin-forbidden transition from singlet $S=0$ to triplet $S=1$ with $\tau_{2}$ is roughly one order of magnitude slower than the allowed singlet-singlet transition with lifetime $\tau_{1}$. This tentative model will be advanced by further time-resolved as well as steady-state studies and quantum chemical calculations on similar complexes in order to identify vibrational marker bands for the assignment of the various redox- and spin-states.

\section{Gas phase: Collision-induced dissociation and photofragmentation studies}

In preparation of gas phase femtosecond time-resolved pump-probe spectroscopy employing photoinduced fragmentation, the ground state fragmentation patterns are investigated. The combination of electrospray ionization mass spectrometry (ESI-MS) with laser photofragmentation allows for a structural and dynamical investigation of isolated gas phase species. The ionic species are produced by the electrospray process from a liquid solution (acetonitrile) and subsequently stored and mass selected with isotopic resolution in a 3D ion trap (Bruker amaZon series). Cpd1 and Cpd2 gave relatively clean mass spectra in the cation mode with singly (and doubly charged) species at $\mathrm{m} / \mathrm{z}$ 547.3 (273.5) and $\mathrm{m} / \mathrm{z} 631.3$ (315.5), respectively. The collision-induced dissociation (CID) of these cations resulted in the neutral loss of a Cat or Sq group (identical m/z 220) and in parallel the loss of a methyl (m/z 15) and tert-butyl group (m/z 57) as fragmentation channels (Figure 2 (left, top). The dioxolene loss is the major channel for the Sq, whereas for the Cat the methyl loss dominates. We recorded also CID fragment yield curves in order to elucidate the binding energies (activation energies for dissociation) of the Sq vs. the Cat unit. Clearly, the activation energy for dissociation for the cation of Cpd2 is larger than that for the cation of Cpd1 (Figure 2, (bottom)). Hence, we conclude the Sq being the more stable species. From the interrogation of the stored species with a ns- 
IR laser we have obtained infrared multiple photon dissociation (IRMPD) spectra of the two different Co-complexes in the $\mathrm{C}-\mathrm{H}$ stretching region (Figure 2 (right)).
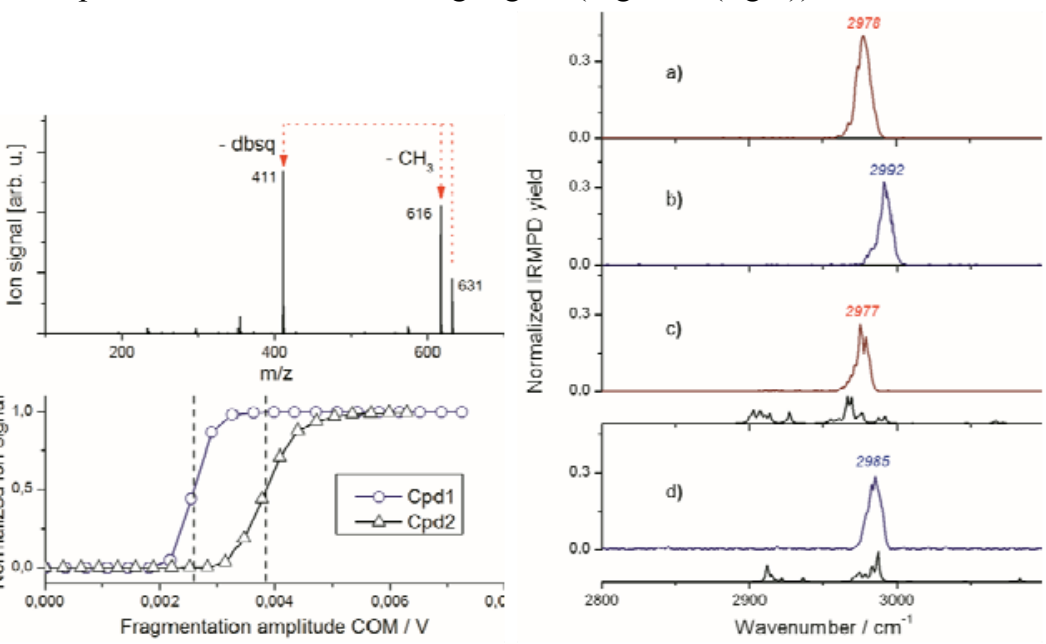

Fig. 2. Left: Top: Electrospray ionization mass spectrum of $\mathbf{C p d} 2$ with indication of fragmentation channels. Bottom: Fragment yield curves for collision-induced dissociation of the cations of $\mathbf{C p d} 2$ and Cpd2. Fragmentation voltage converted to center-of-mass (COM) values. Right: IRMPD spectra of the electrospray ionized a), c) singly and b), d) doubly charged cations of a)-b) (Cpd1) and c)-d) (Cpd2) complexes. Black/lower trace in c) and d): spectra obtained by the DFT/B3LYP/cc-pVTZ, scaled by 0.96 .

The fragmentation channels resulting from collision or IR induced dissociation are the same. The spectra (normalized total fragment yield as a function of laser frequency) are very sparse and only one band was observed. The ground states of the $\mathrm{Sq}$ and the Cat are not distinguishable from this band. A clear blue shift by $10-20 \mathrm{~cm}^{-1}$ upon increase of the charge state was detected. DFT calculations reproduce the band structure and blue shift reasonably well. These IR fragmentation studies, in particular the determination of the ground state fragmentation channels, branching ratios and product yields for Sq vs. Cat complexes are a prerequisite for femtosecond pump-probe studies under the same mass-spectrometric conditions for electronic excited states. The goal being to compare electronic dynamics of SCO and VT compounds under different solvation conditions (gas phase vs. solution).

\section{Acknowledgements}

Deutsche Forschungsgemeinschaft SFB-/TRR 88 (www.3met.de). HJK gratefully acknowledges financial support by Deutsche Forschungsgemeinschaft Priority Program "Molecular Magnetism", SPP 1137. YN gratefully acknowledges Carl-Zeiss-Stiftung for financial support.

\section{References}

1. J-F. Letard, P. Guionneau, L. Goux-Capes, Top. Curr. Chem. 235, 221 (2004)

2. M. Graf, G. Wolmershäuser, H. Kelm, S. Demeschko, F. Meyer, H.-J.Krüger, Angew. Chem., Int. Ed. 49, 950 (2010)

3. M.M.N. Wolf, R. Gross, C. Schumann, J. A. Wolny, V. Schünemann, A. Dossing, H. Paulsen, J. J. McGarvey, R. Diller, Phys. Chem. Chem. Phys. 10, 4264 (2008)

4. P. Tourón Touceda, S. Mosquera Vázquez, M. Lima, A. Lapini, P. Foggi, A. Dei, R. Righini, PCCP 14, 1038 (2012)

5. A. Bhunia, M. T. Gamer, L. Ungur, L. F. Chibotaru, A. K. Powell, Y. Lan, P. W. Roesky, F. Menges, C. Riehn, G. Niedner-Schatteburg, Inorg. Chem. (2012) DOI:10.1021/ic300065x 